

\section{TÁCTICA Y ESTRATEGIA}

Mi táctica es mirarte aprender como sos quererte como sos.

Mi táctica es hablarte y escucharte construir con palabras un puente indestructible.

Mi táctica es quedarme en tu recuerdo no sé cómo ni sé con qué pretexto pero quedarme en vos.

Mi táctica es ser franco y saber que sos franca y que no nos vendamos simulacros para que entre los dos no haya telón ni abismos.

Mi estrategia es en cambio más profunda y más simple.

Mi estrategia es que un día cualquiera no sé cómo ni sé con qué pretexto por fin me necesites. 


\title{
8. Aspectos relacionados a la educación sexual y reproductiva de las jóvenes migrantes de Baja California, México
}

\author{
Humberto González Galbán ${ }^{1}$ \\ DOI: https://doi.org/10.5377/pdac.v16i0.10232 \\ Recibido: 29/05/2020 - Aceptado: 29/06/2020
}

\begin{abstract}
Resumen: La realización del artículo tiene como objetivo conocer aspectos sobre educación sexual y reproductiva de la población inmigrante joven residente en la región fronteriza del norte de México específicamente, Baja California, un Estado atractivo para las jóvenes migrantes del país por su situación geográfica limítrofe con Estados Unidos. Metodológicamente se ha analizado diferentes fuentes documentales relacionadas a la salud sexual y reproductiva de las jóvenes migrantes a Baja California. Se consultó la Encuesta de Salud Reproductiva en México de la Secretaría de Salud 2003 -ESRMEX-, Encuesta de Salud Reproductiva en la Adolescencia, 2006 -ESRABC- del Colegio de La Frontera Norte (2006), así como registros estadísticos de instituciones como el INEGI.
\end{abstract}

Algunos de los hallazgos encontrados se refieren al escaso conocimiento sexual de las jóvenes lo que parece no atenuar las enfermedades de trasmisión sexual y el alto valor de embarazos no planificados en las mismas. Los más importantes informantes sobre la educación sexual y reproductiva son los padres y maestros y no los pares de las jóvenes o los medios de comunicación como se suponía, no obstante, se requiere un mayor conocimiento de dichos temas a través de la comunicación filiar e institucional como escuelas y centros de salud. Se resalta que las políticas dirigidas a resolver las problemáticas de salud reproductiva de la juventud, no son totalmente efectivas si buscan incidir sólo en el conocimiento y el uso de anticonceptivos de los adolescentes sin tratar de trabajar en la resolución de contradicciones esenciales existentes que impiden el desarrollo pleno de los jóvenes, particularmente de segmentos poblacionales de gran peso relativo en contextos como el de Baja California, como los inmigrantes. La extensión de actitudes permisivas en torno a la sexualidad de las más jóvenes, pero sin dotar a las mismas de los conocimientos necesarios para que a partir de ello no experimenten una gestación no deseada, se agudiza en las inmigrantes, aspecto que se hace evidente en el trabajo.

Palabras Claves: Educación sexual y reproductiva, jóvenes migrantes, Baja California, México.

\section{Aspects related to sexual and reproductive education of young migrants from Baja California, Mexico}

Abstract: The purpose of the article is to find out about sexual and reproductive education of the young immigrant population residing in the northern border region of Mexico, such as Baja California, an attractive state for young migrants in the country due to its geographical location bordering the United States. Different documentary sources related to the sexual and reproductive health of young migrants to Baja California have been analyzed methodologically. Among the referred sources are several surveys and population registers that stand out.

Some of the findings found refer to the poor sexual knowledge of young women, which does not seem to mitigate sexually transmitted diseases and the high value of unplanned pregnancies. The most important informants on aspects of sexuality and reproduction are the parents and teachers and not the peers of the young women or the media as it was expected, however, a greater knowledge of these topics was required through filial communication and institutions such as schools and health centers. It is emphasized that the policies aimed at solving the reproductive health problems of youth are effective if they seek to influence the knowledge about sexuality and the use of contraceptives by adolescents without working on the resolution of contradictions that prevent the full development of youth, particularly in population groups such as immigrants. The extension of more permissive attitudes around the sexuality of the youngest, but without endowing them with the necessary knowledge so that from this they do not experience an unwanted pregnancy, which is exacerbated in immigrants, is something that it becomes evident in this work.

Keywords: Sexual and reproductive education, young migrants, Baja California, México.

1 Doctor en Ciencias Sociales, Máster en Estudios de Población por El Colegio de La Frontera Norte en México, Licenciado en Geografía en La Universidad de La Habana. Estudio de posgrado en Universidad Estatal de Moscú, Lomonósov. Actualmente Investigador de El Colegio de la Frontera Norte. e-mail: hggalban@colef.mx 


\section{Introducción}

Un aspecto que amerita ser analizado puesto que puede desarrollar las potencialidades y el aprovechamiento de oportunidades para la juventud, es la existencia de educación dirigida a la salud, pues si bien esta etapa del ciclo de vida suele ser la más saludable para las personas, también en la misma se gestan muchos de los hechos o padecimientos que conducen a situaciones de difícil solución o enfermedades que tendrán que enfrentar en etapas posteriores de su desarrollo como adultos. El comportamiento sexual y reproductivo inadecuado es un claro ejemplo de ello, el cual se ha convertido en un recurrente tópico de investigación, por sus implicaciones sociales, familiares y en el cumplimiento de las aspiraciones de los jóvenes.

Entre los aspectos sociales y familiares de magnitud que se vinculan al comportamiento sexual y reproductivo de los jóvenes destacan la violencia, la drogadicción, la prostitución, el suicidio, la pobreza y la desigualdad que sufren las mujeres, particularmente las migrantes, así como la expulsión de los hogares y escuelas de las adolescentes embarazadas, lo cual en algunos de estos contextos es común y tiene consecuencias poco deseadas para ellas.

Con relación a la salud y la estabilidad emocional de las jóvenes, éstas pueden ser afectadas por relaciones sexuales sin protección lo cual aumenta la probabilidad de un embarazo no deseado o una Infección de Transmisión Sexual (ITS); en Baja California es frecuente, por el temprano inicio de las relaciones sexuales y la exposición a la alta incidencia de ITS, problemática asociada a la conducta sexual y reproductiva de los adolescentes caracterizada por el no uso de preservativos en los primeros "encuentros amorosos", lo que se aprecia particularmente en poblaciones más vulnerables como las migrantes, quienes se encuentran más limitadas al contar con menores redes sociales y familiares que las oriente de manera oportuna.

En Baja California, la edad de inicio de las relaciones sexuales se identifica entre las más tempranas del país y la diferencia entre ésta y el comienzo de alguna unión estable representa intervalos más amplios, lo que genera un mayor tiempo de exposición, y con ello de riesgo a la aparición de un embarazo no planificado, incrementando el potencial de interrupciones provocadas de gestaciones, o embarazos no planificados y/o no deseados. Ello puede haber condicionado que, de los casi 64 mil nacimientos registrados en esta entidad en el año 2015, poco más de 11 mil fueron de madres menores de 20 años y la tasa de fecundidad para estas jóvenes presenta valores de los más elevados de la región con más de cien nacimientos por cada mil mujeres adolescentes existentes, manifestándose además una tendencia al alza, lo que puede generar más nacimientos no planificados o no deseados (INEGI, 2015).

Según la Encuesta Nacional de Salud Reproductiva (2003), -ENSAR- una forma de limitar los problemas reproductivos y las infecciones de transmisión sexual sería no sólo mediante un uso adecuado de los anticonceptivos, sino también de la ampliación y mejoramiento de la educación sexual involucrando a los padres, maestros y médicos (Chávez y Uribe, 2007, pp. 175-190).

Los estados fronterizos mexicanos presentan los más altos niveles de uso de anticonceptivos con una prevalencia del $73.6 \%$, valor superior al alcanzado a nivel nacional, sin embargo, entre los adolescentes de la región que tienen relaciones sexuales este valor es de sólo 44.3\% (ENSAR), pudiendo asociarse a ello la falta de planeación de la relación sexual como atenuante de la "culpa" generada por la censura social aún existente al respecto, o por la forma impulsiva con lo que suelen responder los jóvenes ante algunos estímulos lo que puede conducir a los adolescentes a mantener conductas de riesgo, que puede llevar a un embarazo no deseado o una ITS, situación que podría estar relacionado a una difusión y acceso aún insuficiente, todo lo que evidencia, en última instancia, la necesidad de una adecuada educación sexual para jóvenes y la atención de las instituciones del Estado vinculadas a las problemáticas de las mujeres más jóvenes puesto que aunque los adolescentes tengan conciencia del riesgo, sus conductas priorizan posiblemente primero lo emocional a lo cognitivo (Gaete; 2015, pp. $436-443)^{2}$.

2 Según lo presentado en una revista especializada en Psicología, las investigaciones sobre neurodesarrollo han demostrado que los adolescentes tienen conciencia de los riesgos, pero esto no los inhibe de presentar conductas que los exponen a situaciones que son contrarias a sus potencialidades para alcanzar sus ideales o proyectos futuros en la vida. Ello debido a una maduración cerebral más temprana desde el punto de vista socioemocional que de control cognitivo, lo cual suele presentarse en mayor grado entre los 15 y 17 años de edad. 
Otro aspecto vinculado al embarazo adolescente es la mortalidad infantil. Una investigación realizada mostró implicaciones negativas para la descendencia de las adolescentes, con mayores probabilidades de muerte en las primeras etapas de la vida lo cual se incrementa sustancialmente si la madre además de ser muy joven es migrante (González y Herrera; 2015, p. 28) ${ }^{3}$.

El embarazo en la adolescencia potencia la inestabilidad conyugal, y con ello los conflictos familiares. Algunos investigadores (Salazar et al, 2008, pp. 171-172, y Llanes et al; 2009, pp. 1-7), en similares contextos plantean que las mujeres que tuvieron un hijo en la adolescencia muestran un mayor porcentaje de divorcios. Este hecho podría estar influido, por el matrimonio en la adolescencia que se realiza para legitimar el nacimiento producto de un embarazo prenupcial, pero no para darle una mayor durabilidad a la unión.

Desde el punto de vista sociodemográfico, la conducta sexual y reproductiva de los adolescentes condiciona otros eventos relevantes no previstos, por tanto, generalmente no deseados, como el incremento de la fecundidad adolescente y con ello del crecimiento natural de la población que recibiría más beneficios, al igual que la sociedad, si estuviera ocupada en su formación profesional o técnica y no en funciones reproductivas.

\section{Metodología}

La salud sexual de las jóvenes de la frontera Norte de México presenta características peculiares condicionadas, en buena medida, por la inmigración ya sea del sur del país como de los estados limítrofes norteamericanos. Debido a ello se hace énfasis que ésta se puede clasificar como una investigación documental. Se toma en cuenta en los tópicos aludidos datos aportados por diferentes encuestas, básicamente: la Encuesta de Salud Reproductiva en México de la Secretaría de Salud 2003 -ESRMEX-, Encuesta de Salud Reproductiva en la Adolescencia, 2006 -ESRABC- del Colegio de La Frontera Norte (EL COLEF), así como registros estadísticos de instituciones como el INEGI.

\section{Discusión de resultados}

\subsection{La salud sexual de las jóvenes en Baja California}

Existen grupos poblacionales que están expuestos a sufrir de manera más acentuada las consecuencias de diversos problemas que afectan a la sociedad. Uno de los de mayor peso cuantitativo e importancia estratégica en México, son los jóvenes y entre estos, destaca para el caso del norteño Estado mexicano de Baja California, las mujeres inmigrantes que llegan básicamente del centro y sur del país y se asientan en esta entidad atraídas en gran medida por la demanda de fuerza de trabajo del sector maquilador de exportación (Cruz; 2003, pp. 97-116).

Entre los aspectos que más afecta a las mujeres jóvenes inmigrantes, está el comportamiento sexual en la adolescencia, en este sentido los padres e instituciones como las escuelas y centros médicos, tienen una escasa y mala comunicación con los adolescentes respecto a la temática. En este contexto, los jóvenes llegan a la primera relación sexual con una muy limitada información, lo que condiciona el comportamiento "irresponsable" que a futuro desencadena graves problemas para ellos, sus parejas y familias en general, como las enfermedades de trasmisión sexual y los embarazos no planificados.

Una de las formas básicas para prevenir las infecciones o padecimientos de transmisión sexual (ITS) es conocer de su existencia y las vías de contagio. En el caso de que exista un riesgo de contraer alguna ITS es importante estar informado acerca de los síntomas y el acceso para la realización de pruebas específicas que en el caso de las jóvenes inmigrantes puede presentar mayores limitaciones y por tanto consecuencias negativas en el plano social y de la salud.

Con relación al conocimiento básico que implica al menos haber oído hablar sobre enfermedades o infecciones que pueden ser transmitidas por relaciones sexuales, se apreció que está generalizado, sin grandes diferencias entre el ser una joven nacida en la entidad o una inmigrante; sin embargo para las últimas, el desconocimiento de los síntomas por medio de los que se puede manifestar una ITS en las mujeres, sobrepasa a las nativas $(11.4 \%$ vs. $6.0 \%$, respec-

3 La mortalidad de las personas en las etapas más temprana de la misma depende, en gran medida, de las condiciones de vida en que se desarrollan, referidas a la atención médica prenatal de la madre así como del hijo luego de su nacimiento, a las condiciones sanitarias, de nutrición y del entorno familiar, social y cultural (Bronfman, 2003: 205-220), aspectos ante los que diversas subpoblaciones se encuentran en situaciones desventajosas, entre éstos se destacan las jóvenes inmigrantes (Molina; 2002). 
tivamente) -ESRBC, 2006-. Además, las jóvenes nacidas fuera de la entidad mostraron mayores limitaciones para reconocer que un hombre posee una enfermedad sexual, ello se registró en el $18.3 \%$ de las inmigrantes mientras para las nacidas en la entidad la proporción fue del $12.3 \%$ lo que incrementa el riesgo en las primeras (EL COLEF; 2006).

En cuanto a las vías mediante las que se puede evitar el contagio de alguna ITS, incluyendo el VIH-SIDA, alrededor de tres quintas partes de las jóvenes está consciente de la importancia del condón, ello es más notorio entre las inmigrantes, mientras que menos de una tercera parte de las jóvenes hace referencia a la no promiscuidad en las relaciones de pareja. Sin embargo, la generalidad de las jóvenes que tienen vida sexualmente activa no asume que su pareja le podría transmitir el VIH-SIDA o alguna otra ITS, depositando la confianza en un comportamiento no promiscuo de su compañero; casi cuatro de cada cinco jóvenes no piensan que su pareja pueda transmitirles el VIH-SIDA o alguna otra infección por relaciones sexuales, creencia que sustentan principalmente en la supuesta fidelidad, relegando a un plano marginal la protección con métodos de barrera a estas enfermedades (EL COLEF; 2006).

Según la fuente referida en el anterior párrafo, entre las prácticas de riesgo para la aparición de una ITS en las jóvenes sexualmente activas está el no uso de condones, lo que es más frecuente de lo imaginado, pues a pesar de que una elevada proporción está consciente de dicho riesgo -por las posibles causas que se refirieron anteriormente-, alrededor de tres cuartas partes de ellas, o no los usan nunca, o solo lo hacen algunas veces, situación que se agudiza entre las migrantes. Es destacable también que son las casadas o unidas consensualmente quienes en mayor grado sustituyen el preservativo por la confianza de una pareja estable.

Coincidentemente con lo planteado otros autores que abordan problemáticas de género vinculadas a las jóvenes hacen referencia el hecho de que una parte importante de las jóvenes, se abstienen de pedirle a sus parejas que usen condones en sus relaciones sexuales (Colombo, Pombo y Luxardo, 2012, pp. 161-182:); en este caso, en alrededor de la mitad de las adolescentes más jóvenes -con menos de 18 años-, particularmente las inmigrantes, se presenta dicha situación -ENSAR .2006-.
Otra razón por la cual muchas jóvenes no piden el uso de preservativo a sus parejas es porque consideraban que no existía ningún riesgo o porque no habían planeado las relaciones ${ }^{4}$, argumentaciones que difieren de cuando sí se les solicitó el uso a algunas parejas, destacándose en este caso las situaciones de relaciones casuales o con personas de mayor edad que la joven (González; 2013, pp. 131-152), también representó un incentivo importante para el uso de condones el temor a un embarazo, particularmente en el caso de las inmigrantes.

Derivado de la situación anteriormente planteada, se manifiesta una mayor incidencia de ITS entre las inmigrantes que en las residentes nativas de Baja California puesto que carecen en mayor grado de una adecuada información, un factor que incide en la elevada proporción de casos lo que también representa un mayor riesgo de embarazos no planificados en la adolescencia.

En el caso particular del VIH-SIDA, las fuentes de información señalan a México como uno de los países de América Latina con más número de casos de este padecimiento, destacándose al respecto la región de la frontera norte, donde se ha concentrado una parte importante de los mismos desde finales de la década de los noventa hasta años recientes, situación que ha estado acompañada por una más elevada letalidad en estados como Baja California, donde para el año 2004 la tasa de mortalidad por VIH-SIDA se duplicó con respecto a la del nivel nacional, continuando con el tercer lugar al respecto, entre todas las entidades del país (Zapata , González y Rangel; 2012, pp. 191-208).

Varios son los aspectos que pueden estar incidiendo en la manifestación de esta problemática, particularmente en los jóvenes inmigrantes. El temor a ser relegado socialmente propicia que quienes se perciben en riesgo de estar infectados no acudan a realizarse la prueba de detección y, por lo tanto, no cuenten con un diagnóstico oportuno que les permita atenderse antes de desarrollar la sintomatología propia de la enfermedad lo cual inmoviliza la prevención por cuanto no se aceptará la posibilidad de infección con alguien que no se conozca que es seropositivo y no tenga el estereotipo que se le atribuye a quienes sí lo son (ONUSIDA, OPS y UNICEF, 2009).

Además, en el caso de las personas ya diagnosticadas con VIH-SIDA conlleva a que por temor a que se

4 Otra causa dada para el no uso de anticonceptivos es el deseo de embarazarse lo que es algo poco esperado en regiones más urbanizadas como es la que estamos analizando. 
descubra socialmente su estado serológico, aumente en ellas la ansiedad y el estrés, el aislamiento y el sufrimiento, evadan la atención médica e incluso el tratamiento, contribuyendo a un empeoramiento de su estado de salud (González, Iglesias, Mena y Bojórquez; 2013, pp. 399-407).

\subsection{La reproducción sexual de las jóvenes se- gún condición migratoria}

Otro potencial resultado indeseado de una sexualidad ejercida sin la información adecuada, en el plano individual, sería que se presente un embarazo no planificado que represente una interrupción en los proyectos de vida en una etapa que se asume como formativa mediante la educación institucionalizada. También puede incidir en la perpetuación de diferencias de género que afectan a las mujeres, así como la extensión de condiciones de pobreza en la que se forman y desarrollan las familias, problemas que pueden alcanzar mayores dimensiones entre los inmigrantes que no han podido desarrollar un apoyo económico y/o social. En ese sentido, es importante conocer aspectos sobre condicionantes, la magnitud, riesgos o implicaciones de la reproducción intergeneracional de las jóvenes de Baja California, según la situación migratoria de las mismas.

Con relación a información que confirmaría la importante magnitud de la reproducción se observó que la proporción de jóvenes (de 15 a 29 años de edad) residentes en Baja California que han estado embarazadas representa casi a la mitad de las mismas (46.8\%), valor que se elevó más de 10 puntos porcentuales entre las inmigrantes (58.2\%) (EL COLEF; 2006).

La referida situación se presentó más aguda para las adolescentes (de 15 a 19 años de edad) inmigrantes pues la proporción de las mismas que habían estado embarazadas se elevaba el $90 \%$ por encima de las residentes, es decir, casi se duplicaron los embarazos en adolescentes inmigrantes con respecto a los que experimentaron las mujeres de 15 a 19 años de edad nacidas en esta entidad.

Aunque el inicio de las relaciones sexuales de las migrantes es algo más tardío que el de las nativas -para las sexualmente activas- con una media entre 16 y 17 años de edad, respectivamente, el no uso de anticonceptivos en las primeras relaciones incrementa el riesgo de un embarazo en edades juveniles, situación que se presentó en mayor grado entre las inmigrantes en el contexto estatal analizado
(58.1\% vs. $46.7 \%$ ) -ESRBC, 2006- lo cual confirmaría un supuesto de que la primera relación sexual se presenta en más alto nivel de forma imprevista o no planeada, bajo la creencia de que no se podría embarazar en este primer encuentro, lo que posiblemente denota la poca trascendencia que las jóvenes conceden a sus posibles implicaciones.

En cierta medida también se hacen patentes problemáticas de género al no atreverse a pedir a su pareja usar preservativo o practicar el coito interrumpido para prevenir un posible embarazo, así como de limitaciones en el conocimiento sobre el uso adecuado de métodos anticonceptivos. Es destacable también que entre los inmigrantes una parte de las jóvenes buscaban un embarazo, esta es una práctica común en zonas rurales de alta marginación del país como estrategia para acceder a la maternidad y/o asegurar una pareja.

\subsection{Atención médica durante el embarazo, parto y posparto}

Resulta relevante para la salud reproductiva de las jóvenes y de sus hijos la atención médica que reciben durante el proceso del embarazo, misma que ha alcanzado una considerable cobertura en las zonas urbanas de México (Chávez y Uribe, P.; 2007, pp. 175-190), así como en la generalidad de Baja California donde sólo se registró 3.2\% -ENSRM, 2003de las jóvenes que no recibieron alguna revisión prenatal.

La condición migratoria parece estar relacionada a un comportamiento diferencial, al superar en 50 por ciento la proporción de inmigrantes a las nativas que no fueron revisadas durante el embarazo, llamando la atención con relación a ello que mientras la mayor parte de estas últimas expusieron ante ello el sentirse bien, no considerando necesario el contar con el servicio médico, de las migrantes que no recibieron el referido servicio una parte declaró también que ello fue por falta de dinero o por problemas de acceso a los servicios médicos (EL COLEF; 2006).

Al igual que la revisión prenatal, la atención durante el parto resulta importante para la salud de las mujeres y su descendencia. Este servicio es recibido básicamente en establecimientos del Seguro Social, en clínicas y hospitales privados. Otra peculiaridad de la región, es que poco más de una de cada 20 jóvenes oriundas de Baja California residentes en este Estado dan a luz en los Estados Unidos, con lo que se busca, en general, que el niño adquiera una 
serie de ventajas por el nacimiento en el vecino país del norte.

Durante el período de puerperio - o de cuarentena$78 \%$ de las jóvenes residentes de Baja California recibieron alguna consulta médica. Durante el período previo al parto resultaron menos favorecidas las inmigrantes que superaron en más de un $50 \%$ a las adolescentes del Estado que no se realizaron alguna consulta médica (ENSRM, 2003). A pesar de lo necesario de estas visitas al médico, ello está condicionado, en buena medida, al tipo de parto experimentado. Así, por ejemplo, en el caso de que el parto ocurriera a través de una cesárea, la mujer debería concurrir a la consulta en mayor frecuencia que el caso de un parto normal.

Otra de las implicaciones de importancia sería la realización de un aborto ante un embarazo no planificado. A pesar de las limitaciones de información entorno a las interrupciones de embarazo en la entidad, es posible asumir que la mayor parte de éstas pueden representar un riesgo para la salud y hasta para la vida, por la ilegalidad que pesa sobre esta práctica en Baja California al igual que en la generalidad del país, lo que obliga a las jóvenes con menos recursos económicos a recurrir a abortos clandestinos en condiciones sanitarias inadecuadas. Estudios realizados muestran el paso de mujeres a las cercanas ciudades norteamericanas para realizarse abortos (Ojeda, 2004, pp. 133-144), dado que en el vecino país esta práctica no es ilegal, pero implica un costo económico que muchas jóvenes, posiblemente en mayor grado las migrantes, no puedan cubrir.

De acuerdo con los datos disponibles en la Encuesta de Salud Reproductiva en la Adolescencia de Baja California (2006), los medios para la realización de los abortos fueron principalmente "algún medicamento" para las nacidas en la entidad y los "tés" para las migrantes, lo cual es una práctica precolonial en México que les fue trasmitida a las jóvenes por las madres y no por las amigas.

Un detalle relevante en el contexto de la interrupción de los embarazos son las consecuencias posteriores. Entre las menos dotadas de recursos económicos y de información, como es el caso de las inmigrantes, de las que poco más de la mitad, expresaron que tuvieron problemas de salud o emocionales, asociados a la realización de un aborto provocado en condiciones no propicias (González, H.; 2013, pp. 131-152). En sentido contrario, es- tudios realizados por la Organización Mundial de la Salud (OMS; 2012) dan a conocer que una interrupción de embarazo con la asistencia de los especialistas médicos, fármacos adecuados y de condiciones higiénicas propicias, no implica mayor riesgo para la mujer.

Como condicionantes de afectaciones de la salud por la realización de un aborto, se deberían agregar las presiones sociales y religiosas, lo cual puede ser inferido a partir de que, entre los problemas de salud que plantean haber tenido las inmigrantes, vinculados al haberse practicado un aborto, se encuentran básicamente los de tipo emocional tales como depresión, tristeza y culpa. (González H.; 2013, pp. 131-152).

En Baja California, la educación sexual tiene como fin contrarrestar la falta de información, los perjuicios y la confusión en la toma de decisiones de los jóvenes, por lo que es asumida por instituciones que consideran básicamente dos modelos como los centros educativos y en otros el Institucional de Salud y Planificación Familiar, en los que se adoptan diferentes estrategias que deben tomar en cuenta las necesidades reales de los jóvenes, así como la sensibilización de distintos sectores sociales y gubernamentales acerca de la importancia de educar sexualmente a los adolescentes (Aguilar, Santos, Díaz Infante, Mejía, Vernon y Martín; 2008, pp. 14-69).

El modelo escolar formal se sustenta en textos y conferencias de los profesores, que tienen un carácter informativo en aspectos básicamente biológicos y reproductivos, los que se encuentran insertos en el plan de educación básica. También, se puede considerar la implementación de lo antes señalado con la estrategia educativa recreativa a través de la que los jóvenes tienen la posibilidad de cubrir necesidades como puede ser la de contar con ciertos conocimientos mediante talleres de sexualidad (Aguilar, Santos, Díaz Infante, Mejía, Vernon, y Martín; 2008, pp. 14-69).

En el modelo Institucional de Salud y Planificación Familiar se incluyen programas que llevan a cabo instituciones como la Secretaría de Salud, el Instituto Mexicano del Seguro Social, la Dirección Integral de la Familia (DIF) y el programa Fundación Mexicana para la Planificación Familiar (MEXFAM), entre otras. También se desarrollan estrategias comunitarias que hacen hincapié en las condiciones de vida con iguales derechos para los hombres y 
las mujeres, con modalidades como servicios clínicos para el embarazo adolescente, consultorio médico general, entre otros. (Aguilar, Santos, Díaz Infante, Mejía, Vernon, y Martín; 2008, pp. 14-69).

A pesar de la diversidad de estrategias empleadas, se ha evidenciado que éstas no han sido totalmente adecuadas. Así vemos que los medios por los cuales las jóvenes tuvieron las primeras informaciones sobre diferentes aspectos relativos a la sexualidad y reproducción (Gráfico $N^{\circ} 1$ ), como son las relaciones sexuales, los métodos anticonceptivos (Gráfico $\mathrm{N}^{\circ} 2$ ), el VIH-SIDA (Gráfico $\mathrm{N}^{\circ} 3$ ) y los embarazos no deseados, fueron los maestros y los padres, luego con algún protagonismo al respecto aparecen los médicos, y en un plano muy marginal las amigas y los medios televisivos. Entre las diferencias destaca la muy alta proporción de migrantes, con relación a las nativas, a quienes nadie les habló sobre dichas temáticas.

\section{Gráfico $\mathrm{N}^{\circ} \mathbf{1}$ Baja California: primera persona 0} medio que sirvió como fuente de información a la

\section{jóvenes en temas de educación sexual}

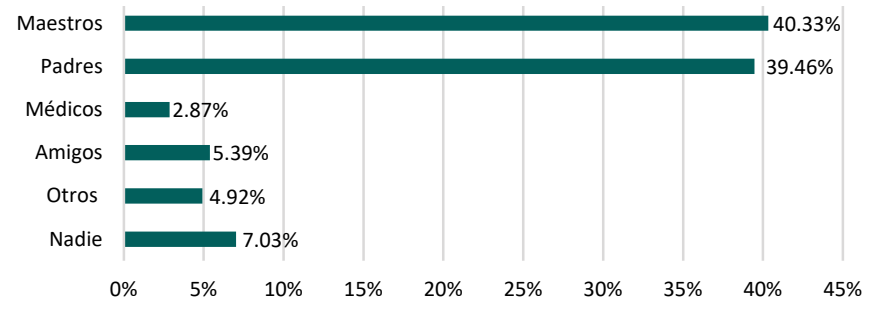

Fuente: elaboración propia a partir de datos de la Encuesta de Salud Reproductiva en la Adolescencia, Baja California, 2006.

Gráfico No2 Baja California: primera persona 0 medio que sirvió como fuente de información sobre métodos anticonceptivos a las jóvenes

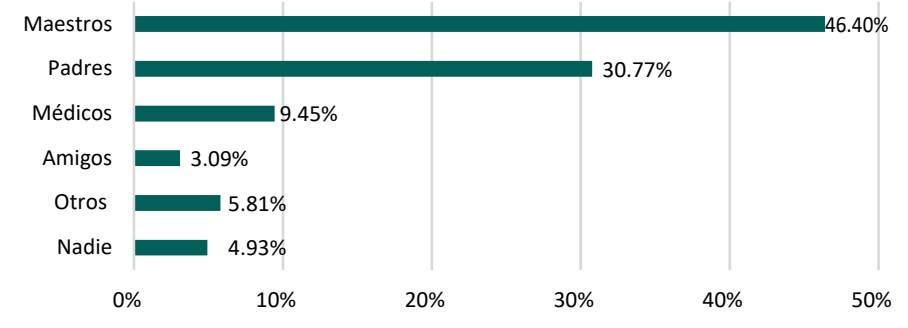

Fuente: elaboración propia a partir de datos de la Encuesta de Salud Reproductiva en la Adolescencia, Baja California, 2006.
Gráfico N`3 Baja California: primera persona 0 medio que sirvió como fuente de información a las jóvenes sobre VIH-SIDA

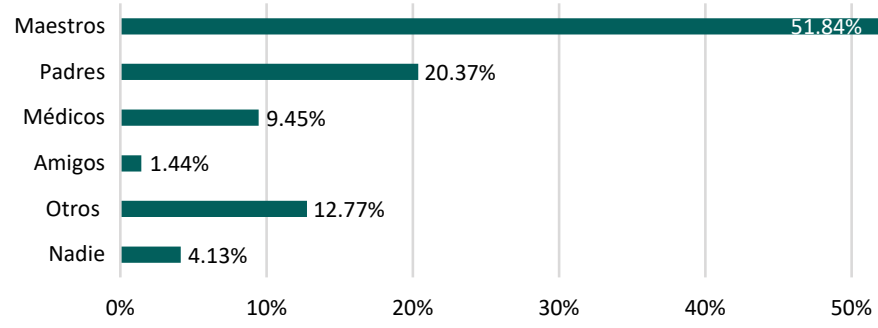

Fuente: elaboración propia a partir de datos de la Encuesta de Salud Reproductiva en la Adolescencia, Baja California, 2006.

Gráfico N4 Baja California: primera persona 0 medio que sirvió como fuente de información sobre embarazos no deseados a las jóvenes

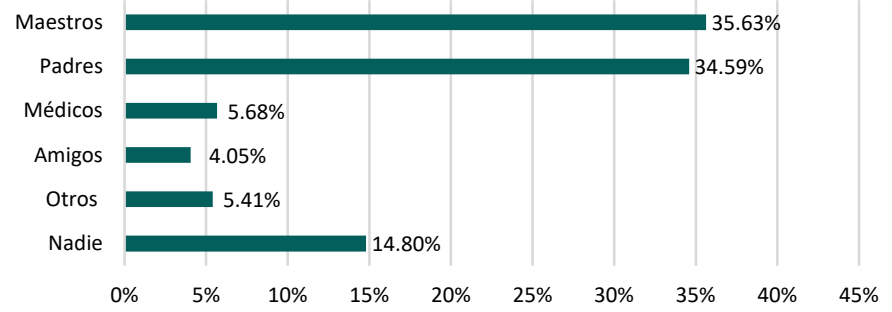

Fuente: elaboración propia a partir de datos de la Encuesta de Salud Reproductiva en la Adolescencia, Baja California, 2006.

Otra limitación destacable es el hecho de que los temas de salud sexual y reproductiva se abordan superficialmente o con escaso dominio del tema por parte de las personas o medios relacionados a estas funciones para que se profundice la participación de los médicos para conocimiento del VIH-SIDA en particular, esto hace incrementar la enseñanza de la sexualidad en el plano filial e institucional de la escuela y los centros médicos.

Las instituciones de la comunidad y el Estado deben recibir la ayuda de diferentes especialistas disciplinarios para que se hagan efectivos los programas que en este caso son de trascendente importancia para las jóvenes, incluyendo el caso que aquí nos ocupa; las migrantes (González, H; 2013). Las limitaciones de las redes de apoyo familiar y social que inciden en la manifestación de diversas problemáticas sociales, de igual manera en el acceso a información adecuada sobre el tema sugiere la necesidad de difundir más estas importantes temáticas entre los inmigrantes. 
Es necesario señalar que, para lograr la efectividad de los programas de educación sexual, se debe buscar la asistencia coordinada de especialistas de diferentes disciplinas en todos los niveles de organizaciones comunitarias y gubernamentales a fin de que dichos programas alcancen efectividad y lleguen a la totalidad de los jóvenes, incluyendo a los jóvenes inmigrantes.

Vinculado a lo antes señalado, se hace necesario destacar que en el marco de los programas de educación sexual se deben desarrollar proyectos dirigidos al análisis y resolución de las contradicciones esenciales que afectan a la adolescencia y la juventud, sustentados en el criterio de que ello es necesario para que los más jóvenes puedan asumir responsablemente los cambios en su sexualidad. No resulta suficiente tratar de incidir directamente en el conocimiento que sobre las relaciones íntimas y el uso de anticonceptivos pueda tener este segmento de la población.

\subsection{Implicaciones del embarazo no planificado para las jóvenes y sus familiares}

Conjuntamente con la magnitud de los embarazos juveniles y sus posibles eventos resultantes, como la interrupción de un embarazo, puede ser de interés conocer las implicaciones a corto plazo de las gestaciones tempranas, cuando éstas se llevan a término, lo cual está asociado con los cambios estructurales y funcionales en los hogares que pueden estar afectando de manera más aguda a los jóvenes inmigrantes.

Ello se relaciona con diversos aspectos vinculados con la reproducción entre los que se destaca el incremento de los nacimientos de niños fuera del matrimonio, el porcentaje de madres jóvenes que no se han casado ni unido consensualmente, la proporción de madres divorciadas, el número de hijos viviendo solo con uno de sus progenitores y la disminución del tiempo de vida adulta compartida con los cónyuges y sus hijos.

La deserción escolar en el Estado de Baja California, es una de las problemáticas que más preocupan a las autoridades educacionales, padres y sociedad en general, lo cual puede estar condicionado por varios factores, entre los que se destaca el que se presente un embarazo. En este sentido, se cuenta con la información de que alrededor de la sexta parte de las jóvenes se encontraba estudiando cuando experimentaron el primer embarazo, proporción que resultó mayor para las nacidas en la entidad, de estas fueron las migrantes las que en mayor grado dejaron de estudiar cuando se presentó la gestación. La causa de ello pudiera encontrarse en la mayor precariedad en las condiciones de vida de las migrantes, así como en las menores redes sociales de apoyo, particularmente las redes familiares de estas últimas (EL COLEF; 2006).

Otra consecuencia común ante un primer embarazo es el abandono -presumiblemente involuntario- del hogar paterno. La información disponible al respecto muestra que una parte importante de las jóvenes residían con sus padres cuando se embarazaron $(41.8 \%)$ y de éstas dos de cada cinco se vieron obligadas a abandonar la casa de la familia. Dicha situación afectó de manera particular a los inmigrantes, lo que presumiblemente esté vinculado a menores posibilidades materiales y de espacio existente en sus lugares de residencia, por lo que se haría más difícil la aceptación de nuevos integrantes familiares (González; 2013, pp. 131-152).

Por otra parte, la fecundidad adolescente es un tema relevante en la agenda pública del país, ya que la maternidad a temprana edad se relaciona con un más elevado riesgo para la salud física y mental de la mujer. Entre las complicaciones obstétricas se encuentran la hipertensión inducida por el embarazo, la anemia ferropriva y el aborto realizado en condiciones inadecuadas. Si la joven lleva a tiempo el embarazo se exponen a infecciones, hemorragias por desprendimiento prematuro de la placenta normoincerta, alteraciones en la dinámica uterina, prolongación del trabajo del parto por poca cooperación de la paciente a consecuencia de la inmadurez y escasa información sobre el evento, lo que puede influir en el incremento de los partos instrumentados o cesárea (Peláez, 1997, p. 72).

Otro aspecto de interés está referido a lo que plantea Cancino -1997- acerca de que las investigaciones sobre el embarazo adolescente se centran más en riesgos médicos que en sus efectos socio-psicológicos, a pesar de ser estos últimos más comunes para la madre y el hijo que los efectos biológicos, (Cancino; 1997, p. 72), por lo que se requiere de más investigación al respecto.

Otra implicación de trascendencia el referido a la mortalidad infantil. Con relación a ello investigaciones realizadas (González y Herrera; 2015, p. 28) evidencian que la mortalidad de los hijos afecta en mayor medida a las madres jóvenes inmigrantes que las 
que son nacidas en Baja California. La diferencia en valores de indicadores al respecto como es la tasa de mortalidad infantil, fue estimada aproximadamente en siete muertes más, por cada mil nacidos vivos para los inmigrantes de esta entidad, en el período 1988-1997 (Molina; 2002, pp. 11-22). En años posteriores, aunque los niveles de mortalidad han disminuido, las diferencias desfavorables con respecto a los inmigrantes no parecen haber cambiado. Según datos del año 2006, las madres que han sufrido la pérdida de algún hijo entre los inmigrantes supera en 50 puntos porcentuales a las madres originarias del Estado que se encuentran en igual situación, manteniéndose para finales de la primera década del actual siglo una menor probabilidad de sobrevivencia de los hijos de madres migrantes (González y Herrera; 2015, p. 28).

La vulnerabilidad de los infantes a morir en los primeros meses o años de vida, asociado a la inmigración, en cierta medida se relaciona con las limitaciones que experimentan las personas que se desplazan de una zona a otra para acceder a la riqueza económica o social existente en el lugar de destino, lo que determina las condiciones de vida de estos, afectando de manera directa la salud de las madres y las condiciones de sobrevivencia de sus hijos menores.

No obstante lo antes señalado, varios autores han planteado que tiene mayor trascendencia para más agudas afectaciones en la salud materno-infantil las mayores limitaciones de información sobre aspectos reproductivos que las condiciones socioeconómicas de subsistencia (Von Glascoe, González y Guendelman; 1995, p. 13), lo que pudiera explicar la mayor vulnerabilidad al respecto en las migrantes, particularmente de las que llevan menos tiempo de residencia en el nuevo destino, en este caso en el Estado de Baja California, ya que las mismas cuentan con menos redes sociales de apoyo que las orienten y/o ayuden en el proceso del embarazo, parto y cuidados durante la crianza de los hijos, lo cual es de suma importancia para la supervivencia infantil (Bronfman; 2003: pp. 205-220, Infante; 1990, pp. 419-429).

Además de los aspectos relacionados a la salud reproductiva, en los análisis que se vienen haciendo desde varias décadas sobre el embarazo adolescente vienen desde varias décadas gran número de investigadores (Tolbert y Morris; 1995, pp. 1-15, Prada, Singh y Wulf; 1990, p. 96, Hamel; 1992, pp.
1-32), se ha hecho hincapié en su vinculación con otros problemas que afectan a los adolescentes, las familias y la sociedad en general. Aunque existen discusiones en torno al tipo y sentido de la relación: embarazo adolescente, problemas sociales, la generalidad de los especialistas coinciden en considerar el embarazo en edades tempranas, como un factor que puede limitar las posibilidades de superación profesional y con ello de ascenso social de las jóvenes, por lo que las adolescentes deben contar con las posibilidades de prevenirlo o posponerlo, en lo que la educación al respecto es algo prioritario.

\section{Conclusiones}

Con el presente artículo se ha logrado conjuntar información generada, en buena medida, en el departamento de Estudios de Población de EL COLEF, sobre la educación sexual y reproductiva de las jóvenes inmigrantes de Baja California, lo que ha permitido el desarrollo de análisis de diferentes tópicos de los que existen pocos antecedentes en el ámbito regional y nacional, resultando ello de utilidad en investigaciones sociales que tomen en cuenta de forma directa o indirecta aspectos que afecten a una parte significativa de la juventud bajacaliforniana.

Como primer resultado de interés se encuentra el escaso conocimiento que tiene, aunque se ha sido medido en forma elemental, o sea sin profundizar en el mismo, sugirió una cierta universalidad, no obstante, no ha atenuado la incidencia de ITS con mayores afectaciones para el caso de las inmigrantes.

Las jóvenes inician su vida sexual de forma poco informada o sin atender a los riesgos en el embarazo generalmente no planificado en estas edades, el cual alcanza altos valores en Baja California, destacándose entre sus condicionantes la extensión de actitudes más permisivas en torno a la sexualidad de las más jóvenes ${ }^{5}$, pero sin dotar a las mismas de los conocimientos necesarios para que a partir de ello no experimenten una gestación no deseada. Así el no uso de anticonceptivos efectivos o su utilización inadecuada, particularmente en las primeras relaciones sexuales completas, propicia embarazos, especialmente en poblaciones con ciertas características como son el ser más joven o el ser inmigrante (González; 2013, pp. 131-152).

De acuerdo a lo planteado en investigaciones reali-

5 Ello puede estar condicionado a la cercanía e intensa interrelación con los Estados Unidos, lo que puede haber favorecido la transmisión de normas socioculturales menos conservadoras en torno a las relaciones sexuales de las jóvenes. 
zadas (Von Glascoe, C; González, M. A. y Gueldelman, S.1995, p. 13), es más importante para la salud sexual y reproductiva de las jóvenes la información que ellas tengan sobre estas problemáticas que las destacadas condiciones materiales de subsistencia.

Es revelador que los principales agentes de las primeras informaciones recibidas por las jóvenes sobre diferentes aspectos de la sexualidad y reproducción en Baja California son los maestros y las madres y no los pares o medios de comunicación como sería posible suponer. Así mismo llama la atención la vinculación que se puede establecer entre la carencia de redes y la relativamente elevada proporción de jóvenes inmigrantes a quienes nadie les habló al respecto. En este sentido, ante la percepción de lo limitado de dicha información, las jóvenes expresaron el deseo de recibir más conocimiento sobre dichos temas mediante una mayor comunicación filiar (madre) e institucional por medio de las escuelas y los centros médicos.

Un aumento acelerado de nacimientos provenientes de las mujeres jóvenes, o al menos de un decrecimiento más lento de dicha variable para la referida población, en Baja California, puede tener trascendentes implicaciones en el área sociodemográfica, al funcionar como freno o hasta como agente de inflexión en el descenso general de la fecundidad regional, incentivando el crecimiento demográfico, pero también potenciando los embarazos no planificados.

Los embarazos no deseados o no planificados, que afectan más a las jóvenes inmigrantes que a las nacidas en la región, traen aparejados, en general, trascendentes problemáticas sociales y familiares que requieren ser solucionados, básicamente mediante la educación sexual y reproductiva, así como el acceso a un sistema de salud especializado eficiente.

Resulta oportuno resaltar que las políticas dirigidas a resolver las problemáticas de salud reproductiva de la juventud, no son totalmente efectivas si sólo buscan incidir de forma directa en el conocimiento sobre la sexualidad y el uso de anticonceptivos de los adolescentes sin tratar de trabajar en la resolución de contradicciones esenciales existentes que impiden el desarrollo pleno de los jóvenes, particularmente de segmentos poblacionales de gran peso relativo en contextos como el de Baja California, como los inmigrantes.

La aceptación de afirmaciones de que, en condicio- nes homogéneamente propicias tales como un estado de salud físico y psicosocial adecuado, un mayor nivel educacional (que incluya la educación sexual y reproductiva), una buena nutrición, acceso a los sistemas de salud y de planificación y protección familiar, entre otros aspectos, se reducen los riesgos del embarazo y limitarían, en buena medida, las preocupaciones médicas a los estratos poblacionales con menos posibilidades sociales y sanitarias como pueden ser las adolescentes inmigrantes.

\section{Referencias bibliográficas}

- Aguilar, O., Santos, L., Díaz Infante, M., Mejía, R. Vernon, R. y Martín, A. (2008), Guía operativa para programas de educación sexual y servicios de salud reproductiva para jóvenes, Population Council/ Fronteras y MEXFAM, México, D.F. pp. 14-69.

- Bronfman, M., (2003), Como se vive se muere. Familia, redes sociales y muerte infantil. UNAM, Centro Regional de Investigaciones Multidisciplinarias. Cuernavaca, pp. 205-220.

- Cancino, C., (1997), El embarazo en la adolescencia: ¿Un problema?, Compilación de investigaciones sobre salud reproductiva en Cuba. Centro de Estudios Demográficos de Cuba. La Habana, Cuba. Pp. 72.

- Chávez, A. M. y Uribe, P., (2007), La salud reproductiva en México. Análisis de la Encuesta Nacional de Salud Reproductiva 2003. Secretaría de Salud/CRIM/UNAM, México, D.F. pp. 175190.

- Colombo G., Pombo, G. y Luxardo N., (2012), Género, embarazo y adolescencia. Modelos familiares, redes de apoyo y construcción de proyectos personales desde la perspectiva de los adolescentes, Revista Internacional de Investigaciones en Ciencias Sociales, Vol. 8, No.2,. Argentina, pp.161-182.

- El Colegio de la Frontera Norte, (2006). Base de datos de la Encuesta de Salud Reproductiva en la Adolescencia, Baja California, Tijuana, México.

- Cruz, Rodolfo. (2003), "Algunos factores asociados a la participación femenina en los mercados de trabajo: Ciudades de la Frontera Norte y Áreas Metropolitanas de México" en Frontera Norte, Vol.5, No.9, enero- junio, El Colegio de La Frontera Norte, pp. 97-116. 
- Gaete, V., (2015), Desarrollo psicosocial del adolescente, Sociedad Chilena de Pediatría, Revista Chilena de pediatría, Vol.86, Issue 6, pp.436443. https//doi .org/1016/j.rchipe.2015.07.005.

- González, H.; (2013). Problemáticas del comportamiento sexual y reproductivo de los jóvenes inmigrantes de Baja California. Revista Frontera Norte, 25 (50), pp. 131-152.

- González, H. y Herrera, L.; (2015). Mortalidad Infantil y preescolar en el estado de Baja California. Análisis de condicionantes biodemográficos relacionados a la historia reproductiva de la madre, Población y Salud en Mesoamérica. San José, Costa Rica, 12(2), pp. 28.

- González, H., Iglesias, M., Mena, M. y Bojórquez-Chapela, I. R., (2013). "Diseño y aplicación en Cuba de un constructo para identificar dimensiones sociales de la discriminación por $\mathrm{VIH} /$ sida". Salud Publica de México, 55(4), pp. 399-407.

- Hamel, P. (1991). Crónica de un embarazo anunciado: Criterios de riesgos en adolescentes urbanas pobres. Servicio Nacional de la Mujer, Chile, pp. 1-32.

- INEGI, (2015), Registros Administrativos. Natalidad: Conjunto de datos, nacimientos registrados por municipio de residencia habitual según grupos quinquenales de edad de la madre al nacimiento.

- Infante, C. (1990). Utilización de servicios de atención prenatal: Influencia de la morbilidad percibida y de las redes sociales de ayuda. Revista Salud Pública de México, 34(4), pp. 419429. Recuperado de http//www.redalyc.org/ pdf/106/10632405.pdf

- Llanes, A. et al, (2009), Embarazo en la adolescencia. Intervención educativa, Revista Archivo médico de Camaguey, Cuba, pp. 1-7.

- Molina, M. C. (2002). Mortalidad infantil, según estatus migratorio de la madre en los estados de la Frontera Norte de México: Periodo 1987-1997 (Tesis de maestría). El Colegio de la Frontera Norte, Tijuana, B. C, pp. 11-22.

- Ojeda, N. (2004). Silent and alone: Abortion in a transborder context, En E. R. Hansen and D. Mattlingly. Woman and change at the U.S.- Mexico border. The University of Arizona Press, pp. 133-144.
- ONUSIDA, OPS, UNICEF, (2009). Retos planteados por la epidemia de VIH en América Latina y El Caribe 2009.

- http://www.paho.org/hq/dmdocuments/2010/ Retos\%202009\%20espanol\%20final.pdf?ua=1

- OMS, (2012), Abortos sin riesgos: Guía técnica y política para sistemas de salud. Segunda Edición, Capítulo 1.

- Peláez, J., (1997). "Adolescencia. Responsabilidad sexual y prevención en Salud Reproductiva". En Compilación de Investigaciones sobre Salud Reproductiva. Centro de Estudios Demográficos de Cuba. La Habana. Cuba, pp. 72.

- Prada E., Singh, S. y Wulf, D. (1990), Adolescentes hoy, padres del mañana, un perfil de las Américas: Colombia. Alan Guttmacher Institute, Universidad de Texas, pp. 96.

- Salazar, A. et al, (2008), Consecuencias del embarazo adolescente en el estado civil de la madre joven: Estudio piloto en Bogotá, Colombia, Revista Persona y Bioética, Vol.12, No.2. pp. 171-172.

- Tolbert, K. y Morris, K. (1995), "Los hombres y la decisión de abortar. Hacia un modelo de relaciones de género y el aborto". En Coloquio Latinoamericano sobre varones, sexualidad y reproducción. Zacatecas. México, pp. 1-15.

- Von Glascoe, C., González, M. A. y Guendelman, S. (1995). "La salud reproductiva entre mujeres trabajadoras de la industria maquiladora de Tijuana". En conferencia Salud sin Fronteras. México, pp. 13.

- Zapata, R., González, E. y Rangel, G. (2012). Mortalidad por vih/sida en la frontera norte de México: niveles y tendencias recientes, Población y Salud en la Frontera Norte de México (mimeo), El Colegio de la Frontera Norte, Tijuana, México, pp. 191-208. 